\title{
NONLINEAR DYNAMIC BUCKLING OF A COMPRESSED ELASTIC COLUMN*
}

\author{
EDWARD L. REISS \\ (Courant Institute of Mathematical Sciences and New York University) \\ AND \\ BERNARD J. MATKOWSKY \\ (Rensselaer Polytechnic Institute)
}

1. Introduction. A constant, compressive, axial displacement $C$ is applied to each end of a simply supported, slender, elastic column. The constant $2 C$ is called the total end-shortening. The column is given an initial transverse displacement and an initial transverse velocity and we wish to study its resulting motion.

We assume that the column is sufficiently slender that the deformations occur with small strains and small but finite displacements and Hooke's law is valid. We employ a modified dynamic Euler-Bernoulli beam theory in which the axial strain depends nonlinearily on the gradients of the displacements (see, e.g., [1, 2, 3, 4]. It is essentially equivalent to the dynamic von Kármán plate theory for the one-dimensional motion of an infinite strip plate. The effect of damping is included and, as is customary, the axial inertia of the column is neglected.

The linear theory of dynamic stability, which is summarized in Sec. 4, predicts that the unbuckled or straight column is dynamically stable (unstable) if $C<C_{1}\left(C \geq C_{1}\right)$. Here $C_{1}$ is the smallest bifurcation point of the solutions of the nonlinear static (time independent) theory. The static theory is summarized in Sec. 3. Specifically, the solution of the linear dynamic theory grows exponentially in time if $C>C_{1}$. Since this theory is derived by assuming small amplitude motions, it can only be valid for "small" times. Therefore we employ the nonlinear dynamic theory to study the stability of the unbuckled column and its motion for $C>C_{1}$ and all time.

In Sec. 5 we solve the nonlinear dynamic theory for monochromatic initial data and any value of $C>C_{1}$. The amplitude of the initial data is arbitrary. In Sections 6 and 7 we consider the nonlinear dynamic theory for small but otherwise arbitrary quiescent data and values of $C$ slightly greater than $C_{1}$. We obtain a formal asymptotic expansion of the solution by a two time method [5,6]. The use of two time scales in the analysis of other stability problems for nonlinear partial differential equations was first given in $[7,8,9] .{ }^{1}$ One time scale $t$ is required to describe the initial or "fast" behavior and the second or "slow" time $\theta$ is required to describe the long-time, large-amplitude re-

* Received May 10, 1970. The research reported in this paper was supported by the Office of Naval Research, U. S. Navy Contract N00014-67-A-0467-0006, and the National Science Foundation grant GP9453.

1 J. B. Keller and S. Kogelman are currently using related methods to study other stability problems. 
sponse. This method has been applied to other nonlinear dynamic elastic stability problems. We expect to report on these results elsewhere.

The response of the column as approximated by the leading term in the asymptotic expansion consists of a fast-time high-frequency motion, which we call the secondary motion, superposed on a primary motion that depends only on $\theta$. The motion depends strongly on the character of the initial data. Thus for the undamped column the primary motion is periodic and may be either an oscillation about the unbuckled state, a polarized oscillation about one of the two static buckled states, or a swaying oscillation between the two static buckled states. In the last case, if the amplitude of the secondary motion is large compared to the amplitude of the primary motion, the motion of the column would appear as a high-frequency oscillation with a slow drift between the buckled states. When damping is present, the column always approaches one of the static buckled states as $t \rightarrow \infty$. It may sway back and forth a finite number of times before the oscillations finally polarize about and damp to one of the static buckled states.

Previous investigations of other problems of dynamic buckling of columns have been concerned primarily with the response to time-dependent axial forces $[10,11]$ (in which case the problem is linear) and time-dependent axial displacements $[2,3,4,12]$ with only monochromatic initial data.

2. Formulation. The centroidal axis of the column coincides with the segment $[0, L]$ of the $X$ axis, and the cross-section lies in the $Y, Z$ plane. Its area and its moment of inertia with respect to $Z=0$ are denoted respectively by $A$ and $I$. The ends of the column are simply supported. A compressive axial displacement $C$, which is independent of time $T$, is applied to each end. At $T=0$ the column is given a prescribed initial velocity and initial displacement. The subsequent motion is assumed to occur in the $X, Z$ plane. We employ a modified nonlinear, dynamic, Euler-Bernoulli beam theory [1-4] to determine the motion. We denote the $X$ (axial) and $Z$ (transverse) displacements by $U(X, T)$ and $W(X, T)$ respectively and the axial stress and strain by $\sigma(X, t)$ and $e(X, t)$. All other stresses and displacements are neglected in the theory. Then, using subscripts to denote partial derivatives, the beam theory with transverse damping is:

$$
\begin{gathered}
(E I / A) W_{X X X X}-\left(\sigma W_{X}\right)_{X}+\rho W_{T T}+\delta W_{T}=0 \\
\sigma_{X}=0, \\
\sigma / E=e \equiv U_{X}+1 / 2\left(W_{X}^{2}\right), \\
W=W_{X X}=0 \text { for } X=0, L, \quad T \geq 0, \\
U(0, T)=-U(L, T)=C>0, \quad T>0 ; \\
W(X, 0)=F^{*}(X), \quad W_{T}(X, 0)=G^{*}(X), \quad 0<X<L .
\end{gathered}
$$

Eqs. (2.1a) and (2.1b) are the $Z$ and $X$ equations of motion and (2.1c) is a statement of Hooke's law and the strain-displacement relation. In (2.1b) we have made the usual assumption that the axial inerita force is negligible. The positive constants $E$, $\rho$ and $\delta$ are, respectively, Young's modulus, the density, and the damping coefficient. If $\delta=0$ in (2.1a) then there is no damping. The prescribed initial data $F^{*}(X)$ and $G^{*}(X)$ are assumed to satisfy (2.2a) at $X=0, L$. Since (2.1b) implies that $\sigma=\sigma(T)$, we integrate (2.1c) from $X=0$ to $X=L$ and use (2.2b) to obtain 


$$
\sigma(T) / E=-2 C / L+(1 / 2 L) \int_{0}^{L} W_{x}^{2} d X
$$

We define the following dimensionless variables:

$$
\begin{gathered}
x \equiv X / L, \quad t \equiv T / r, \quad w(x, t) \equiv W(X, T) / L, \quad \lambda(t) \equiv-k \sigma(T) / E, \\
F(x) \equiv F^{*}(X) / L, \quad G(x) \equiv G^{*}(X) r / L,
\end{gathered}
$$

and the dimensionless parameters

$$
k=L^{2} A / I, \quad r^{2} \equiv \rho L^{2} k / E, \quad \Gamma \equiv\left(\delta L^{2} k\right) /(2 E r), \quad c=C / L>0 .
$$

Then, inserting (2.5) into (2.1a), (2.2a), (2.3) and (2.4) and using (2.1b), we obtain:

$$
\begin{gathered}
w_{x x x}+\lambda v_{x x}+w_{t t}+2 \Gamma w_{t}=0, \quad 0<x<1, \quad t>0 . \\
\lambda=2 c k-(k / 2) \int_{0}^{1} w_{x}^{2} d x, \quad t>0 ; \\
w=w_{x x}=0 \text { for } x=0,1 \quad \text { and } t>0 ; \\
w(x, 0)=F(x), \quad w_{t}(x, 0)=G(x), \quad 0 \leq x \leq 1 .
\end{gathered}
$$

The nonlinear dynamic problem, which we call Problem $N$, is to determine a sufficiently smooth function $w(x, t)$ in the region $D:\{x, t \mid t \geq 0,0 \leq x \leq 1\}$ that satisfies the nonlinear integro-differential equation (2.6a) and the boundary and initial conditions (2.7) and (2.8). Here $\lambda$ is a nonlinear functional of $v_{x}$ defined by (2.6b). Existence and uniqueness theorems for Problem $N$ with $\Gamma=0$ are stated in [13].

3. The static theory. The nonlinear static theory, which we call Problem $\mathrm{S}$, is to determine time-independent solutions $w(x)$ of (2.6) and (2.7). Since the solutions of Problem S have been analyzed in [14] and elsewhere, we need only summarize the results. The unbuckled state,

$$
w \equiv 0, \quad \lambda=2 c k,
$$

is a solution of Problem S for all values of $c$. It is the only solution of Problem $\mathrm{S}$ if

$$
\lambda \neq \lambda_{n} \equiv(n \pi)^{2}, \quad n=1,2, \cdots .
$$

If $\lambda=\lambda_{n}$ then Problem $\mathrm{S}$ has the solutions

$$
w(x)=A_{n} \phi_{n}(x), \quad \phi_{n}(x) \equiv\left(2^{1 / 2}\right) \sin \left(\lambda_{n}^{1 / 2} x\right),
$$

where for $n=1,2, \cdots$ the amplitudes $A_{n}$ and $c$ are related by

$$
c=c_{n}\left[1+k A_{n}^{2} / 2\right], \quad c_{n} \equiv \lambda_{n} /(2 k),
$$

and $\phi_{n}(x), n=1,2, \cdots$ are normalized eigenfunctions, i.e.

$$
\int_{0}^{1} \phi_{n}^{2}(x) d x=1
$$

The quantities $c_{n}$ are called the critical end-shortenings. A sketch of the static "response," (3.4), is given in Fig. 1.

For each $n$, (3.4) has real (buckled) solutions $A_{n}(c)$ if and only if $c \geq c_{n}$. The solutions branch in pairs from the unbuckled state $A_{n}=0$ at $c=c_{n}$; i.e., the column can buckle in either the $+Z$ or $-Z$ directions. They exist for all $c>c_{n}$ and $A_{n}(c)$ is a monotone in- 


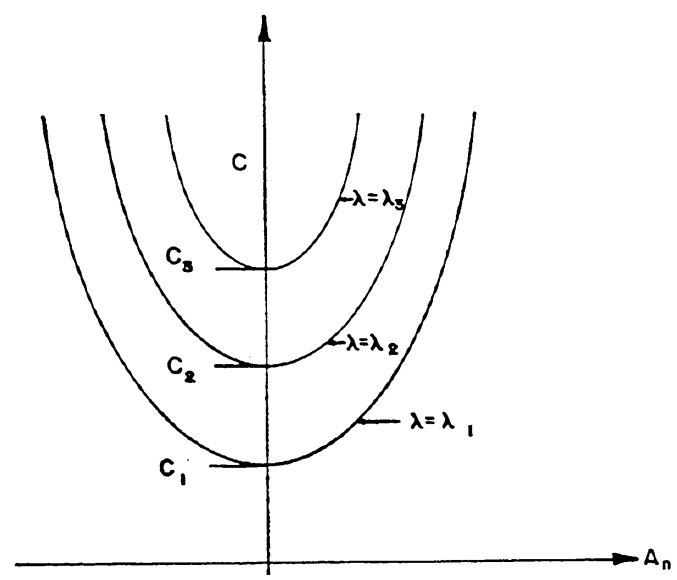

FIG. 1. A sketch of the nonlinear static response of the column. The critical end-shortenings $c_{n}$ are the bifurcation points of the solutions of the nonlinear static theory.

creasing function of $c$. For $c<c_{1}$, (3.1) is the only solution of Problem S. For any $c$ in the interval $c_{n}<c<c_{n+1}$, there are exactly $2 n+1$ solutions. They are the $2 n$ buckled states and the unbuckled state. Let $V_{m}(c)$ denote the potential energy of the buckled states corresponding to $A_{m}$. It is shown in [14] that for fixed $c$ in the interval $c_{n}<c<c_{n+1}$ the energies of the equilibrium states are ordered according to $V_{1}<V_{2}<\cdots<V_{n}<V_{\infty}$, where $V_{\infty}$ is the energy of the unbuckled state.

A possible static criterion for buckling [14] is that at a bifurcation point $c_{n}$, the state with least energy actually occurs. Thus at $c=c_{1}$, the column deforms into one of the two buckled states branching from $c_{1}$ and it remains in that state for $c>c_{1}$.

4. The linear dynamic theory. The linear dynamic theory is conventionally employed to test the stability of the unbuckled state with respect to small initial disturbances. Thus we assume that there is a small parameter $\epsilon$ such that the initial data (2.8) are given by

$$
F(x)=\epsilon f(x), \quad G(x)=\epsilon g(x), \text { for } 0 \leq x \leq 1,
$$

where $f$ and $g$ are independent of $\epsilon$. For small-amplitude motions we substitute

$$
w(x, t)=\epsilon v(x, t)
$$

in (2.6) $-(2.8)$ and linearize the resulting equations with respect to $\epsilon$. This yields the linear dynamic theory:

$$
\begin{gathered}
v_{x x x x}+\lambda v_{x x}+v_{t t}+2 \Gamma v_{t}=0, \text { for } 0<x<1 \text { and } t>0, \\
\lambda=2 c k, \\
v=v_{x x}=0 \quad \text { for } x=0,1 \text { and } t \geq 0, \\
v(x, 0)=f(x), \quad v_{t}(x, 0)=g(x), \text { for } 0<x<1 .
\end{gathered}
$$

We assume that $f$ and $g$ are sufficiently smooth that

$$
f(x)=\sum_{n=1}^{\infty} f_{n} \phi_{n}(x), \quad g(x)=\sum_{n=1}^{\infty} g_{n} \phi_{n}(x),
$$


where $f_{n}$ and $g_{n}, n=1,2, \cdots$, are the Fourier coefficients of $f$ and $g$. The formal solution of (4.3) and (4.4) is

$$
v(x, t)=\sum_{p=1}^{\infty} S_{p}(t) \phi_{p}(x),
$$

where for $\lambda \neq \lambda_{D}$ and $p=1,2, \cdots, S_{D}$ are given by

$$
\begin{aligned}
S_{p}(t) & =H_{p}^{+} \exp \left(\sigma_{p}^{+} t\right)+H_{p}^{-} \exp \left(\sigma_{p}^{-} t\right), \\
H_{p}^{ \pm} & \equiv \mp\left[\frac{\sigma_{p}^{\mp} f_{p} \pm g_{p}}{\sigma_{p}^{+}-\sigma_{p}^{-}}\right], \quad \sigma_{p}^{ \pm} \equiv-\Gamma \pm\left[\Gamma^{2}-\lambda_{p}\left(\lambda_{p}-\lambda\right)\right]^{1 / 2} .
\end{aligned}
$$

For $\lambda=\lambda_{p}$ and $p=1,2, \cdots$ they are given by

$$
\begin{aligned}
S_{\mathcal{D}}(t) & =f_{\mathcal{D}}+\left(g_{\mathcal{D}} / 2 \Gamma\right)[1-\exp (-2 \Gamma t)], \quad \Gamma>0, \\
& =f_{\mathcal{D}}+g_{\mathcal{D}} t ; \quad \Gamma=0 .
\end{aligned}
$$

If $c<c_{p}$ and hence by (3.4) and (4.3) $\lambda<\lambda_{p}$, then (4.5b) implies that $\operatorname{Re} \sigma_{p}^{ \pm} \leq 0$ and thus $S_{p}(t)$ is bounded for all $t>0$. If $c>c_{p}$ then (4.5b) implies that $S_{p}(t) \rightarrow \infty$ exponentially as $t \rightarrow \infty$ unless $H_{p}^{+}=0$. If $H_{p}^{+}=0$ then $S_{p}(t) \rightarrow 0$ as $t \rightarrow \infty$. Finally, if $c=c_{p}$ and $\Gamma>0$ then $S_{p}(t)$ is bounded for all $t$ while if $\Gamma=0$ then $S_{p}(t)$ is bounded (unbounded) if $g_{p}=0\left(g_{p} \neq 0\right)$.

The preceding results suggest the following definitions for the linear dynamic stability of the unbuckled state.

Definition 4.1. The unbuckled state is linearly stable with respect to specific initial data if the solution $v(x, t)$ of (4.3) with that data is bounded in $D$.

Definition 4.2. The unbuckled state is linearly stable if $v$ is bounded in $D$ for arbitrary initial data.

Definition 4.3. The linear stability of the unbuckled state is strong (weak) if $\lim _{t \rightarrow \infty} v=0(\neq 0)$.

Definition 4.4. The unbuckled state is linearly unstable if it is not linearly stable.

Therefore the unbuckled state is linearly stable if $c<c_{1}$ and the stability is strong (weak) if $\Gamma>0(=0)$. If $c$ is in the interval $c_{n}<c<c_{n+1}$ for some $n \geq 1$ and if the initial data satisfy

$$
\sum_{i=1}^{n}\left(H_{i}^{+}\right)^{2}=0
$$

then the unbuckled state is linearly stable with respect to that data. The stability is strong if $\Gamma>0$ and weak if $\Gamma=0$. Since $v(x, t)$ is unbounded as $t \rightarrow \infty$ for all initial data that violate (4.6), the unbuckled state is linearly unstable for all $c>c_{1}$.

Since the linear dynamic theory is presumably valid only for small-amplitude motions, linear instability need not imply that the solutions of the nonlinear dynamic problem are unbounded. Therefore in the remainder of the paper we shall study Problem N.

5. The nonlinear dynamic theory: monochromatic data. If the initial data (2.8) is monochromatic, i.e. if there is an integer $n>0$ and constants $F_{n}$ and $G_{n}$ such that for $0 \leq x \leq 1$

$$
F(x)=F_{n} \phi_{n}(x), \quad G(x)=G_{n} \phi_{n}(x),
$$

then the solution of Problem N, (2.6)-(2.8), is given $b^{2}$

${ }^{2}$ This has been observed previously by Woinowsky-Krieger [2] and others for Problem $\mathrm{N}$ with $\Gamma=0$. 


$$
w(x, t)=B(t) \phi_{n}(x) .
$$

Here $B(t)$ is a solution of the initial value problem

$$
\begin{aligned}
B^{\prime \prime}+2 \Gamma B^{\prime}+K_{n}\left(\beta_{n} B+B^{3}\right) & =0, \quad \beta_{n} \equiv \frac{4}{\lambda_{n}}\left(c_{n}-c\right), \quad K_{n} \equiv k \lambda_{n}^{2} / 2 \\
B(0) & =F_{n}, \quad B^{\prime}(0)=G_{n},
\end{aligned}
$$

where we use a prime to denote differentiation with respect to $t$. In the remainder of this section we shall omit, for simplicity, the subscripts on $K_{n}, \beta_{n}, F_{n}$ and $G_{n}$. An initial value problem similar to (5.3) has been obtained previously by Stoker [15] in a rigid rod and spring model of dynamic column buckling, i.e. a one-dimensional model.

We first consider the undamped column, i.e. $\Gamma=0$. An exact solution of (5.3) can be obtained in terms of elliptic functions. However it is more convenient to examine the phase plane to determine the qualitative behavior. The following discussion partially parallels that in [15]. Thus, in the usual way, we multiply (5.3a) by $B^{\prime}$ and integrate it from 0 to $t$ to obtain

$$
V^{2}+K\left(\beta B^{2}+B^{4} / 2\right)=H \equiv G^{2}+K\left[\beta F^{2}+F^{4} / 2\right] .
$$

Here $V$ is defined by

$$
V=B^{\prime}
$$

The initial conditions (5.3b) were used to evaluate the integration constant $H$ in (5.4). It follows from (5.4) and (5.5) that the initial value problem (5.3) is equivalent to the initial value problem

$$
\frac{d V}{d B}=-\frac{K\left(\beta B+B^{3}\right)}{V}, \quad V(F)=G
$$

in the phase plane.

If $c \leq c_{n}$, i.e. $\beta \geq 0$, then $B=V=0$ (the unbuckled state) is the only singular point of (5.6) and it is a center. Since the integral curves (5.4) of (5.6) are closed in the phase plane (see Fig. 2), $B(t)$ is a periodic function. Thus for $c \leq c_{n}$ the column oscillates periodically about the unbuckled state. This result and subsequent results suggest the following definitions for the nonlinear dynamic stability of the unbuckled state.

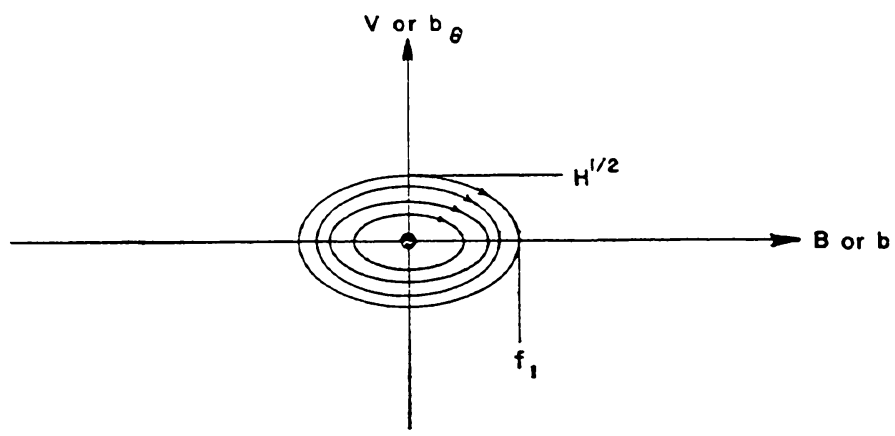

FIG. 2. Phase plane diagram to describe the motion of the undamped column with monochromatic data (5.1) and $c \leq c_{n}$. The origin is the only singular point and it is a center. This figure also represents the $\left(b_{\theta}, b\right)$ phase plane for the primary motion of the undamped column with noisy initial data $(\mu \geq 0)$. 


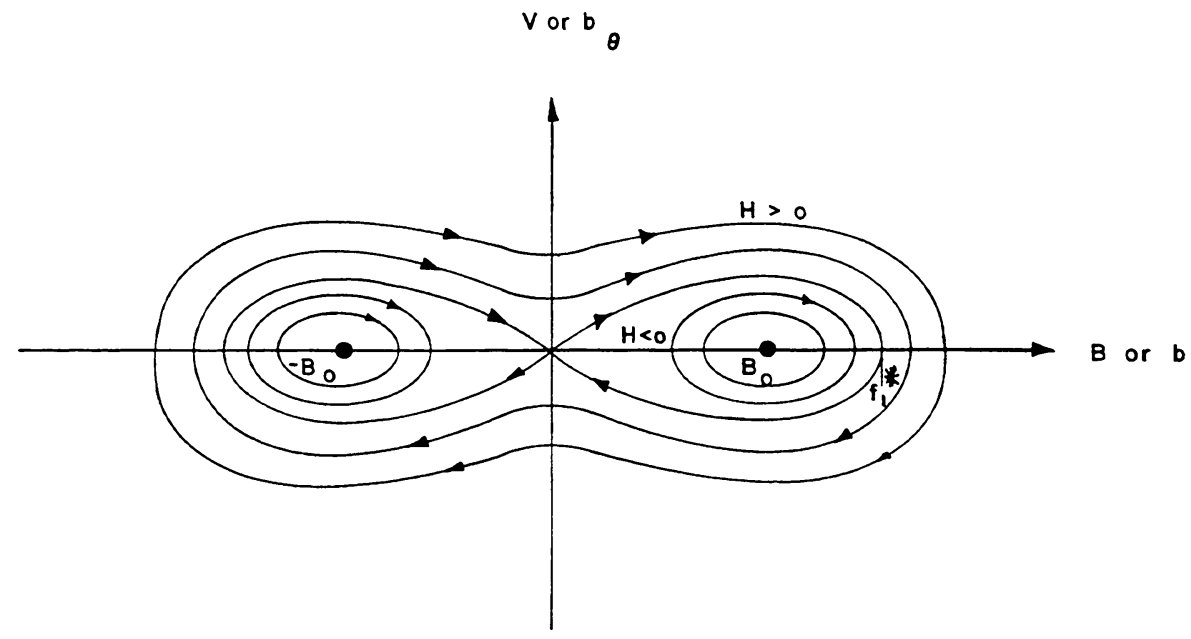

Fig. 3. Phase plane diagram to describe the motion of the undamped column with monochromatic data (5.1) and $c>c_{n}$. The static equilibrium states correspond to the singular point $B=V=0$ (the unbuckled state) and $B= \pm B_{0}, V=0$ (the buckled states). The quantity $H$ is defined in (5.4) and is a measure of total initial energy. The motion is either in the polarized mode $(H<0)$ or the swaying mode $(H>0)$. The figure also represents the $\left(b_{\theta}, b\right)$ phase plane for the primary motion of the undamped column with quiet initial data $(\mu<0)$.

Definition 5.1. The unbuckled state is nonlinearly stable with respect to specific initial data if the solution $w(x, t)$ of Problem $\mathrm{N}$ with that data is bounded in $D$, and if for every $t_{0}>0$ there exists a $t^{*}>t_{0}$ such that $w\left(x, t^{*}\right) \equiv 0$. Here we permit $t^{*}=\infty$.

Thus, the column either passes through the unbuckled state infinitely often or it decays monotonically to the unbuckled state for sufficiently large $t$. We emphasize that this is not required in Definition 4.2 of linear stability.

Definition 5.2. The unbuckled state is nonlinearly stable if it satisfies Definition 5.1 for arbitrary initial data.

Definition 5.3. The nonlinear stability of the unbuckled state is strong (weak) if $\lim _{t \rightarrow \infty} w(x, t)=0(\neq 0)$.

Definition 5.4. The unbuckled state is nonlinearly unstable if it is not nonlinearly stable.

Hence the unbuckled state is linearly and nonlinearly stable with respect to the initial data (5.1) if $c \leq c_{n}$ and the stability is weak.

If $c>c_{n}$, i.e. $\beta<0$, then the three singular points of (5.6) are

$$
\begin{gathered}
B=V=0 ; \\
B= \pm B_{0}, \quad V=0, \text { where } B_{0} \equiv(-\beta)^{1 / 2} .
\end{gathered}
$$

The singular point (5.7a) is a saddle and the singular points $(5.7 \mathrm{~b})$ are centers. The origin (5.7a) corresponds to the unbuckled state and the points (5.7b) correspond to the two static buckled states that branch from $c=c_{n}$. A sketch of (5.4) is given in Fig. 3 for fixed $K$ and $\beta$ and a sequence of $H$ values. The curve with $H=0$, which is usually called the separatrix, passes through the origin. If $H<0$, then for fixed $K$ and $\beta$ (5.4) has two branches of closed curves. Each branch contains one of the singular points (5.7b). Thus for $H<0, B(t)$ is a periodic function of one sign. The column osc - 
lates periodically about one of the static buckled states that branch from $c=c_{n}$. We refer to this motion as the polarized mode of vibration. Therefore if $H<0$ the unbuckled state is nonlinearly unstable according to Definition 5.4. We recall that the unbuckled state is linearly unstable if $H_{n}^{+} \neq 0$ and it has strong linear stability with respect to the monochromatic data (5.1) if $H_{n}^{+}=0$.

If $n>1$ then the static buckled states that branch from $c_{n}$ are unstable according to the static energy criterion (see Sec. 3). However, they are dynamically stable with respect to the data (5.1) in the sense that the solution of Problem $N$ is polarized about a static buckled state. They need not be dynamically stable for arbitrary data.

For $c>c_{n}$ and for each $H>0,(5.4)$ gives a closed curve that contains the three singular points (5.7). Hence $B(t)$ is a periodic function that changes sign twice in each period. Thus the column sways between a neighborhood of each of the static buckled states that branch from $c_{n}$ and passes twice in each period through the unbuckled state. We refer to this motion of the column as the swaying mode. Therefore the unbuckled state has weak nonlinear stability with respect to the data (5.1) if $c>c_{n}$. The nonlinear stability of the unbuckled state thus depends on the sign of $H$. If $H=0$ then $\lim _{t \rightarrow \infty} w(x, t)=0$ and the unbuckled state has strong nonlinear stability with respect to the data (5.1) (we take $t^{*}=\infty$ in Definition 5.1). If $F$ and $G$ are such that $H>0$, then the column will oscillate in the swaying mode.

If the column is damped, $\Gamma>0$, then the first-order initial value problem equivalent to $(5.3)$ is

$$
\frac{d V}{d B}=-\frac{\Gamma-K\left(\beta B+B^{3}\right)}{V}, \quad V\left(F^{\prime}\right)=G .
$$

The origin, (5.7a), is the only singular point of (5.8) when $c \leq c_{n}(\beta>0)$. It is a stable spiral (stable node) if $\Gamma^{2}-4 K \beta<0(>0)$. Then the unbuckled state has strong nonlinear stability with respect to the data (5.1).

When $c>c_{n}$ then (5.8) has the three singular points (5.7). The origin is a saddle and the singularities (5.7b) are stable spirals (stable nodes) if $\Gamma^{2}+8 K \beta<0(\geq 0)$. For simplicity, we shall consider only stable spirals. In Fig. 4 we sketch the "separatrix"; i.e., the solutions of (5.8) that branch from the origin. For any initial data not on the separatrix, the solution of (5.8) or equivalently (5.3) will be "captured" by one of the singular points (5.7b) when $t \rightarrow \infty$. Thus the unbuckled state is nonlinearly unstable. For $|F|$ and $|G|$ sufficiently small, the motion is polarized about one of the static buckled states for all $t>0$ and is captured by this state as $t \rightarrow \infty$. For $|F|$ and $|G|$ sufficiently large, the motion occurs in the swaying mode for a finite time and then becomes polarized about, and is damped to, one of the static buckled states. The final state need not be the one nearest to the initial point. If the initial point is in an unshaded (shaded) region, then the final state will be the buckled state at $B_{0}\left(-B_{0}\right)$.

In order to study completely the nonlinear dynamic stability of the column it is necessary to consider the more general data

$$
F(x)=\sum_{n=1}^{N} F_{n} \phi_{n}(x), \quad G(x)=\sum_{n=1}^{N} G_{n} \phi_{n}(x),
$$

where $F_{n}$ and $G_{n}$ are the Fourier coefficients of $F$ and $G$. The solution of (2.6)-(2.8) is then given by

$$
w(x, t)=\sum_{n=1}^{N} B_{n}(t) \phi_{n}(x)
$$




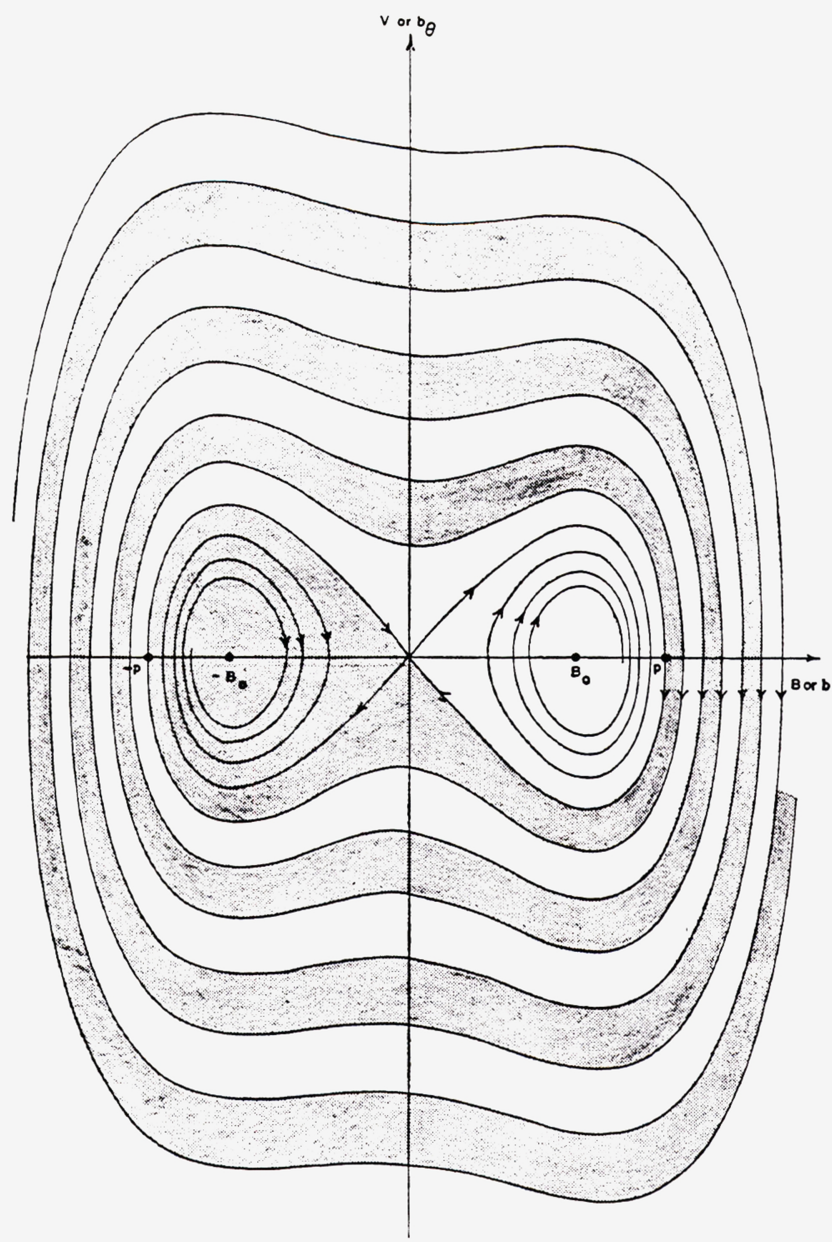

FIG. 4. Phase plane diagram to describe the motion of the damped column with monochromatic data (5.1) and $c>c_{n}$. Representative values of $\Gamma$ and $K$ are employed. The graph was obtained by numerically solving (5.8). The authors are indebted to Dr. L. Bauer for obtaining the numerical results. This figure also represents the $\left(b_{\theta}, b\right)$ phase plane for the primary motion of the damped column with primary (monochromatic) initial data. The shaded and unshaded regions indicate which buckled state will be the final state. If the initial state is in a shaded (unshaded) region, then the final state will be at $-B_{0}\left(B_{0}\right)$.

The functions $B_{n}(t)$ satisfy a system of $N$ coupled, nonlinear ordinary differential equations that cannot be analyzed as readily as (5.3). In the next two sections we study these equations, or equivalently (2.6)-(2.8) for $c$ near $c_{1}$, and small initial data. Although the calculations involved in considering (2.6)-(2.8) are essentially equivalent to those for the system of ordinary differential equations, we prefer to consider the former formulation. It is then easier to interpret the physical meaning of the results, and further it indicates how to employ our procedure for other problems where it is not possible to obtain an equivalent system of ordinary differential equations.

6. The nonlinear dynamic theory: small "arbitrary" data. We consider Problem 
$\mathrm{N}$ with the small-amplitude data (4.1) and with $c-c_{1}>0$ and small. Thus we define the small parameter $\epsilon>0$ by $^{3}$

$$
c=c_{1}+\epsilon^{2},
$$

and for simplicity we assume that the initial data is quiescent; i.e., that there is an integer $N>0$ such that ${ }^{4}$

$$
f(x)=\sum_{n=1}^{N} f_{n} \phi_{n}(x), \quad g(x)=\sum_{n=1}^{N} g_{n} \phi_{n}(x) .
$$

The restriction to quiescent data may not be of practical importance for smooth data since the error made in truncating the series for a numerical evaluation will be small. However, it may be essential for the beam theory (2.6)-(2.8) to be valid. If the data is not quiescent then other small parameters occur. Thus we distinguish between initial disturbances which oscillate rapidly and disturbances which are quiescent, i.e. do not oscillate rapidly. The character of the motion may be significantly different for the two types of data, and hence different asymptotic expansions of the solution may be required. In this paper we consider only quiescent data. Furthermore, when the data are not quiescent it may be necessary to employ a beam theory that includes other effects such as transverse shear deformations. We also assume that the damping is small, that is,

$$
\Gamma=\epsilon \gamma,
$$

where $\gamma$ is independent of $\epsilon$.

We assume that the solution of Problem $\mathrm{N}$ depends essentially on two time scales. One scale is required to describe the small-time behavior, which is approximated by the linear dynamic theory, and the second scale is required to describe the longer-time, large-amplitude response. Thus we define an auxiliary time $\theta$ by

$$
\theta=\epsilon t \text {. }
$$

We seek a formal asymptotic expansion of the solution of Problem $\mathrm{N}$ as $\epsilon$ goes to zero in the form

$$
w(x, t ; \epsilon) \sim \epsilon \sum_{i=0}^{\infty} w^{i}(x, t, \theta) \epsilon^{i} .
$$

The expansion coefficients ${ }^{5} w^{i}(x, t, \theta), j=0,1,2, \cdots$, are assumed to be bounded functions of $x, t$ and $\theta$. They are determined by substituting (6.1)-(6.5) and

$$
\lambda \sim \sum_{i=0}^{\infty} \lambda^{i}(t) \epsilon^{i}
$$

into (2.6)-(2.8) and equating coefficients ${ }^{6}$ of the same powers of $\epsilon$. Thus we obtain for

\footnotetext{
${ }^{3}$ The solution of the static theory motivated the definition (6.1).

4 With only minor modifications, we could consider more general data where $f$ and $g$ depend analytically on $\epsilon$. However, to simplify the presentation we shall omit them from our analysis.

${ }^{5}$ The superscript $j$ in $w^{j}$ is an index.

${ }^{6}$ Alternatively, we could use the two-time method to solve the ordinary differential equations for $B_{n}(t)$ in (5.10). However, we prefer the present approach since it is easier to interpret the results in terms of the physical problem.
} 
$j=0,1,2, \cdots$ :

$$
\begin{gathered}
M w^{i}=r^{j} \equiv-2 w_{i \theta}^{i-1}-2 \gamma w_{t}^{i-1}-w_{\theta \theta}^{j-2}-2 \gamma w_{\theta}^{j-2}-\sum_{k=1}^{j} \lambda^{k} w_{x x}^{i-k}, \\
\lambda^{0}=\lambda_{1} \equiv \pi^{2}, \quad \lambda^{1}=0, \quad \lambda^{2}=2 k-(k / 2) \int_{0}^{1}\left(w_{x}^{0}\right)^{2} d x, \\
\lambda^{i}=-(k / 2) \int_{0}^{1} \sum_{k=0}^{i-2} w_{x}^{k} w_{x}^{i-k-2} d x, \quad j \geq 3 ; \\
w^{i}=w_{x x}^{i}=0 \quad \text { for } \quad x=0,1, \\
w^{i}(x, 0,0)=f(x) \delta_{j 0}, \quad w_{t}^{i}(x, 0,0)=g(x) \delta_{j 0}-w_{\theta}^{i-1}(x, 0,0) .
\end{gathered}
$$

Here $\delta_{j 0}$ is the Kronecker delta, the sum in (6.7) is defined to be zero if $j=0$ and $w^{-1}=$ $w^{-2} \equiv 0$. The linear differential operator $M$ is defined by

$$
M w^{i} \equiv w_{x x x x}^{i}+w_{t t}^{i}+\lambda_{1} w_{x x}^{i}
$$

In obtaining (6.7)-(6.11) we have assumed that termwise differentiation of (6.5) is permissible.

To determine $w^{0}$ we set $j=0$ in (6.7), (6.9) and (6.10). This yields the initial boundary value problem (cf. Eq. 4.3 ):

$$
\begin{aligned}
M w^{0} & =0 ; \\
w^{0}=w_{x x}^{0} & =0, \quad \text { for } \quad x=0,1 ; \\
w^{0}(x, 0,0) & =f(x), \quad w_{t}^{0}(x, 0,0)=g(x) .
\end{aligned}
$$

The formal solution of $(6.12)$ is

$$
w^{0}(x, t, \theta)=\sum_{n=1}^{N} A_{n}^{0}(t, \theta) \phi_{n}(x),
$$

where for each $n, n=1,2, \cdots N$, the coefficients $A_{n}^{0}$ must satisfy

$$
\begin{gathered}
P A_{n}^{0} \equiv A_{n, t}^{0}+\omega_{n}^{2} A_{n}^{0}=0, \\
A_{n}^{0}(0,0)=f_{n}, \quad A_{n, t}^{0}(0,0)=g_{n}, \\
\omega_{n} \equiv\left[\lambda_{n}\left(\lambda_{n}-\lambda_{1}\right)\right]^{1 / 2} .
\end{gathered}
$$

In (6.14) we have used the notation

$$
A_{n, t}^{0} \equiv \frac{\partial A_{n}^{0}}{\partial t}, \quad A_{n, t}^{0} \equiv \frac{\partial^{2} A_{n}^{0}}{\partial t^{2}} .
$$

Since $\omega_{1}=0$, the solution of (6.14a) with $n=1$ is

$$
A_{1}^{0}=a_{1}^{0}(\theta) t+b_{1}^{0}(\theta) \text {. }
$$

where $a_{1}^{0}(\theta)$ and $b_{1}^{0}(\theta)$ are bounded functions to be determined. Since $w^{0}(x, t, \theta)$ is required to be a bounded function of its arguments, we conclude from (6.16) that $a_{1}^{0}(\theta) \equiv 0$. This implies that

$$
A_{1}^{0}=b_{1}^{0}(\theta) \equiv b(\theta) .
$$


The coefficient $A_{1}^{0}$ must also satisfy the initial conditions (6.14b) with $n=1$. Thus we conclude that

$$
\begin{gathered}
b(0)=f_{1}, \\
g_{1}=0 .
\end{gathered}
$$

The condition (6.19) is a restriction on the class of initial data that we can analyze by our method. ${ }^{7}$ If (6.19) is violated then $w^{\circ}$ is unbounded in $t$ as $t \rightarrow \infty$ and a different asymptotic expansion is presumably required to solve Problem $N$ with $g_{1} \neq 0$. Without loss of generality we assume that $f_{1} \neq 0$. The solutions of (6.14) for $n=2,3, \cdots, N$ are

$$
A_{n}^{0}=a_{n}^{0}(\theta) \sin \omega_{n} t+b_{n}^{0}(\theta) \cos \omega_{n} t,
$$

where $a_{n}^{0}$ and $b_{n}^{0}$ satisfy the initial conditions

$$
a_{n}^{0}(0)=g_{n} / \omega_{n}, \quad b_{n}^{0}(0)=f_{n} .
$$

Here $a_{n}^{0}(\theta)$ and $b_{n}^{0}(\theta)$ are bounded functions to be determined. The initial boundary value problem to determine $w^{1}$ is obtained by substituting $j=1$ into (6.7), (6.9) and (6.10). It is given by:

$$
\begin{gathered}
M w^{1}=r^{1}=-2 w_{t \theta}^{0}-2 \gamma w_{t}^{0}=-2 \sum_{n=1}^{N}\left(A_{n, t \theta}^{0}+\gamma A_{n, t}^{0}\right) \phi_{n}(x) \\
w^{1}=w_{x x}^{1}=0, \text { for } x=0,1 \\
w^{1}(x, 0,0)=0, \quad w_{t}^{1}(x, 0,0)=-w_{\theta}^{0}(x, 0,0)=-\sum_{n=1}^{N} A_{n, \theta}^{0}(x, 0,0) \phi_{n}(x),
\end{gathered}
$$

where we have used (6.13). The solution of (6.21) is

$$
w^{1}(x, t, \theta)=\sum_{n=1}^{N} A_{n}^{1}(t, \theta) \phi_{n}(x),
$$

where for $n=1,2, \cdots, N, A_{n}^{1}$ must satisfy

$$
\begin{gathered}
\left.P A_{n}^{1}=R_{n}^{1} \equiv-2 \omega_{n}\left[\left(a_{n, \theta}^{0}+\gamma a_{n}^{0}\right) \cos \omega_{n} t-\left(b_{n, \theta}^{0}+\gamma b_{n}\right) \sin \omega_{n} t\right)\right] \\
A_{n}^{1}(0,0)=0, \quad A_{n, t}^{1}(0,0)=-b_{n, \theta}^{0}(0),
\end{gathered}
$$

where the operator $P$ is defined in (6.14a). Since $\omega_{1}=0$, we obtain from (6.23a) with $n=1$

$$
A_{1}^{1}=a_{1}^{1}(\theta) t+b_{1}^{1}(\theta) .
$$

The boundedness of $A_{1}^{1}$ implies that $a_{1}^{1}(\theta) \equiv 0$ and hence from (6.24)

$$
A_{1}^{1}=b_{1}^{1}(\theta)
$$

From (6.23b) with $n=1$ we conclude that

$$
b_{1}^{1}(0)=0, \quad b_{1, \theta}^{0}(0)=b_{\theta}(0)=0,
$$

where $b_{\theta} \equiv \partial b / \partial \theta$.

7 This is a consequence of the assumed form of the solution (6.5), which is an asymptotic expansion of solutions $w$ which are small, i.e. $O(\epsilon)$. If (6.19) is violated then $w$ is not small, and in the expansion, $w^{0}$ is unbounded as $t \rightarrow \infty$. This is to be expected also from the linear analysis of Sec. 4 (cf. the discussion following (4.5c) with $p=1$ ). 
The solutions of the inhomogeneous problems (6.23) with $n>1$ must be bounded for all $t \geq 0$. Therefore the coefficients of the "resonance-producing" terms in $R_{n}^{1}$ (the right side of (6.23a)) must vanish. This implies that for $n=2,3, \cdots, N$

$$
a_{n, \theta}^{0}+\gamma a_{n}^{0}=b_{n, \theta}^{0}+\gamma b_{n}^{0}=0 .
$$

We solve the first-order ordinary differential equations (6.27) with the initial conditions given in (6.20). This gives

$$
a_{n}^{0}=\left(g_{n} / \omega_{n}\right) e^{-\gamma \theta}, \quad b_{n}^{0}=f_{n} e^{-\gamma \theta}, \quad n=2,3, \cdots .
$$

We insert (6.28), or equivalently (6.27), in $R_{n}^{1}$ in (6.23a). Then, solving (6.23), we obtain

$$
A_{n}^{1}=a_{n}^{1}(\theta) \sin \omega_{n} t+b_{n}^{1}(\theta) \cos \omega_{n} t, \quad a_{n}^{1}(0)=\gamma f_{n} / \omega_{n}, \quad b_{n}^{1}(0)=0
$$

where $a_{n}^{1}(\theta)$ and $b_{n}^{1}(\theta)$ are to be determined. Thus in summary we find from (6.13), (6.17), (6.20) and (6.28) that

$$
w^{0}=b(\theta) \phi_{1}(x)+e^{-r \theta} \sum_{n=2}^{N}\left[\left(g_{n} / \omega_{n}\right) \sin \omega_{n} t+f_{n} \cos \omega_{n} t\right] \phi_{n}(x) .
$$

$w^{1}$ is given by $(6.22),(6.25)$ and (6.29).

To determine $b(\theta)$ we consider (6.7)-(6.10) with $j=2$. Thus $w^{2}$ must satisfy

$$
\begin{aligned}
& M w^{2}=r^{2}=-2 w_{\theta t}^{1}-2 \gamma w_{t}^{1}-w_{\theta \theta}^{0}-2 \gamma w_{\theta}^{0}-\lambda^{2} w_{x x}^{0}, \\
& w^{2}=w_{x x}^{2}=0, \quad \text { for } \quad x=0,1, \\
& w^{2}(x, 0,0)=0, \quad w_{t}^{2}(x, 0,0)=-w_{\theta}^{1}(x, 0,0),
\end{aligned}
$$

where $\lambda^{2}$ is given in (6.8). In $r^{2}$ and the expression for $\lambda^{2}$, we substitute $w^{0}$ given by (6.30) and $w^{1}$. The solution of (6.31) is

$$
w^{2}(x, t, \theta)=\sum_{n=1}^{N} A_{n}^{2}(t, \theta) \phi_{n}(x),
$$

where, for $n=1,2, \cdots N, A_{n}^{2}$ satisfies

$$
P A_{n}^{2}=R_{n}^{2}
$$

and appropriate initial conditions. The inhomogeneous term $R_{n}^{2}$ depends on $b(\theta), a_{n}^{1}(\theta)$ and $b_{n}^{1}(\theta)$. Since it is a lengthy expression we shall not write it down explicitly. From the condition that $A_{1}^{2}$ must be bounded for all $t \geq 0$ we find that $b(\theta)$ must satisfy the nonlinear ordinary differential equation

$$
b_{\theta \theta}+2 \gamma b_{\theta}+K\left(\mu b+b^{3}\right)=0
$$

where $\mu(\theta)$ is defined by

$$
\mu(\theta) \equiv\left(4 / \lambda_{1}\right)\left\{-1+(1 / 8) e^{-2 \gamma \theta} \sum_{n=2}^{N} \lambda_{n}\left[\left(f_{n}\right)^{2}+\left(g_{n} / \omega_{n}\right)^{2}\right]\right\}, \quad K \equiv k \lambda_{1}^{2} / 2 .
$$

We have already shown in (6.18) and (6.26) that $b(\theta)$ satisfies the initial conditions

$$
b(0)=f_{1}, \quad b_{\theta}(0)=0 .
$$

We refer to the differential equation (6.34a) as the amplitude equation and the initial value problem (6.34) as the amplitude problem. Thus $w^{0}$ in (6.30) is completely determined when the amplitude problem is solved. 
Elimination of the resonance-producing terms in $R_{n}^{2}$ for $n \geq 2$ gives equations to determine $a_{n}^{1}$ and $b_{n}^{1}$. To evaluate $b_{1}^{1}$ and hence completely to determine $w^{1}$ we must consider (6.7)-(6.10) with $j=3$. However, we shall not present the results of these calculations and merely consider the approximation given by $w^{0}$.

7. The amplitude problem. We shall first consider the amplitude problem (6.34) with $\gamma=0$ :

$$
\begin{gathered}
b_{\theta \theta}+K\left(\mu b+b^{3}\right)=0, \quad b(0)=f_{1} \neq 0, \quad b_{\theta}(0)=0, \\
\mu=\left(4 / \lambda_{1}\right)\left\{-1+(1 / S) \sum_{n=2}^{N} \lambda_{n}\left[\left(f_{n}\right)^{2}+\left(g_{n} / \omega_{n}\right)^{2}\right]\right\} .
\end{gathered}
$$

Thus $\mu$ is independent of $\theta$ and (7.1) has constant coefficients. The corresponding expression for $w^{0},(6.30)$, with $\gamma=0$ is

$$
w^{0}(x, t, \theta)=b(\theta) \phi_{1}(x)+\sum_{n=2}^{N}\left[g_{n} / \omega_{n} \sin \omega_{n} t+f_{n} \cos \omega_{n} t\right] \phi_{n}(x) .
$$

We refer to the leading term $f_{1} \phi_{1}(x)$ in the initial data (6.2) $\left(g_{1}=0\right)$ as the primary data. The remaining terms in the sums (6.2) are called the secondary data. Since the sum in (7.2) depends only on $f_{n}$ and $g_{n}$ with $n \geq 2$, we refer to $\mu$ as the noise factor. The initial data are defined to be noisy (quiet) if $\mu \geq 0(\mu<0)$. The slow-time standing wave $b(\theta) \phi_{1}(x)$ in (7.3) is called the primary motion. The sum in (7.3) is called the secondary motion. It is the sum of standing waves whose amplitudes are periodic functions of the fast time $t$. We can therefore consider the secondary motion as a high-frequency noise superposed on the slow-time primary motion. Of course, if the amplitude of the secondary motion is sufficiently large then the secondary motion is the main motion and the effect of the primary motion may appear as a slowly varying drift of the main motion.

The amplitude problem (7.1) and (7.2) is the same as (5.3) with $\Gamma=G_{n}=0$ if we identify $\theta, b, K$, and $f_{1}$ in (7.1) respectively with $t, B, K_{n}, \beta_{n}$ and $F_{n}$ in (5.3). Therefore, with the appropriate interpretations, the qualitative discussion of the solution of (5.3) applies to the solution of (7.1). Thus if $\mu \geq 0$ (noisy data) then the origin $b=b_{\theta}=0$ of the phase plane is the only singularity of (7.1) and we obtain the phase plane curves as in Fig. 2. The primary motion is a periodic function of $\theta$ and hence is a slow-time oscillation about the unbuckled state. If $\epsilon$ and the amplitude of the secondary motion are sufficiently small then we conclude, using (6.5) and (7.3) and the definitions in Sec. 5, that the unbuckled state is nonlinearly stable with respect to the data (6.2) and (6.19) if $\mu \geq 0$.

For quiet initial data, $\mu<0$, the phase plane curves are as shown in Fig. 3. If $H<0$, i.e. $\left|f_{1}\right|<f_{1}^{*} \equiv(-2 \mu)^{1 / 2}$, the primary motion is a slow time periodic standing wave. It is polarized about the static buckled state $b=B_{0}$ if $f_{1}>0$ and $b=-B_{0}$ if $f_{1}<0$. If $\epsilon$ and the secondary motion are sufficiently small then, according to the definitions given in Sec. 5 , the unbuckled state is nonlinearly unstable. The linear dynamic theory also predicts that the unbuckled state is unstable. However, if $H>0$, i.e. $\left|f_{1}\right|>f_{1}^{*}$, then the primary motion is a slow-time periodic standing wave in the swaying mode (see Fig. 3). Hence if $\epsilon$ and the amplitude of the secondary motion are sufficiently small, the unbuckled state is nonlinearly stable with respect to the initial data (6.2) (6.19), according to Definition 5.1. If $\left|f_{1}\right|=f_{1}^{*}$, then the primary motion monotonically approaches the unbuckled state as $\theta \rightarrow \infty$.

The amplitude problem (6.34) with $\gamma>0$ and only primary initial data is: 


$$
\begin{aligned}
b_{\theta \theta}+2 \gamma b_{\theta}+K\left(\mu b+b^{3}\right) & =0 ; \quad b(0)=f_{1}, \quad b_{\theta}(0)=0 ; \\
\mu & \equiv-4 / \lambda_{1} .
\end{aligned}
$$

Since the initial data are primary (monochromatic) there is no secondary motion and $w^{0}$ is then given by

$$
w^{0}(x, t, \theta)=b(\theta) \phi_{1}(x) .
$$

It is independent of the fast time. With appropriate changes in notation, (7.4) is equivalent to (5.3) with $n=1, G_{1}=0$ and $\beta_{1}<0$. Thus the discussion given in Sec. 5 (see also Fig. 4) is applicable to (7.4) and (7.5). However for (7.4) the initial point is always on the $b$ axis. Thus the points $\pm p$ on the $b$ axis are critical values of the initial data $f_{1}$. If $\left|f_{1}\right|<p$ then the motion of the column is polarized and damped to the buckled state nearest $f_{1}$. If $\left|f_{1}\right|>p$ the column will sway back and forth a finite number of times passing through the two buckled states. It will eventually be captured by one of these states. The final state need not be the one nearest $f_{1}$. If $f_{1}$ lies in an unshaded (shaded) region then the final state will be the buckled state at $B_{0}\left(-B_{0}\right)$. If $f_{1}$ lies on a boundary between the shaded and unshaded region, i.e. on the separatrix, then the column approaches the unbuckled state as $t \rightarrow \infty$.

For arbitrary initial data satisfying (6.19) and $\gamma>0$, the secondary motion in (6.30) is a slowly damped superposition of fast-time periodic standing waves. For sufficiently large $\theta, \mu(\theta)<0$, see $(6.34 \mathrm{~b})$. Therefore for large $\theta$ the qualitative behavior of (6.34) is similar to that of (7.4). A limited numerical study of (6.34), which is not described here, suggests that this is true for all $\theta$. Depending on the magnitude of $f_{1}$ the primary motion $b(\theta)$ will be, for small $\theta$, either in the polarized mode or in the swaying mode. For suffciently large $\theta$ the primary motion will be in the polarized mode and $\lim _{\theta \rightarrow \infty} b(\theta)= \pm B_{0}$. We wish to emphasize that the final static state need not be the one attained by the solution of (7.4) with the same value of $f_{1}$. Since the secondary motion is also damped, $w^{0}$ will approach a static buckled state as $t \rightarrow \infty$. Thus the unbuckled state is nonlinearly unstable for $c$ satisfying (6.1). Each of the static buckled states branching from $c_{1}$ is also nonlinearly unstable, even though these states are linearly stable, i.e. stable to "infinitesimal perturbations". However, the static buckled states considered as a pair are dynamically stable in the sense that for sufficiently small $\epsilon$ and the initial data satisfying (6.19), the solution of Problem $N$ always approaches one of then as $t \rightarrow \infty$ for $c$ satisfying (6.1).

\section{REFERENCES}

[1] E. Mettler, Dynamic buckling, Handbook of engineering mechanics, McGraw-Hill, New York, 1962, chapter 62

[2] S. Woinowsky-Krieger, The effect of an axial force on the vibration of hinged bars, J. Appl. Mech. 17 $35-36$ (1950)

[3] N. J. Hoff, The dynamics of the buckling of elastic columns, J. Appl. Mech. 18, 68-74 (1951)

[4] N. J. Hoff, Dynamic stability of structures, Proceedings of an international conference on dynamic stability of structures, Pergamon Press, New York, 1967

[5] J. D. Cole, Perturbation methods in applied mathematics, Blaisdell, Waltham, Mass., 1968

[6] E. L. Reiss, On multi-variable asymptotic expansions, SIAM Rev. (to appear)

[7] B. J. Matkowsky, Asymptotic solution of a nonlinear stability problem, Studies in Appl. Math., no. 5; a collection of papers presented at the Conference on Qualitative Theory of Nonlinear Differential and Integral Equations (Madison, Wis., 1968), SIAM, Philadelphia, Pa., 1969. 
[8] B. J. Matkowsky, Nonlinear dynamic stability: A formal theory, SIAM J. Appl. Math. 18, 872-883 (1970)

[9] B. J. Matkowsky, A simple nonlinear dynamic stability problem, Bull. Amer. Math. Soc. 17, 620$625(1970)$

[10] S. Lubkin and J. J. Stoker, Stability of columns and strings under periodically varying forces, Quart. Appl. Math. 1, 215-236 (1943)

[11] V. V. Bolotin, The dynamic stability of elastic systems, Holden-Day, San Francisco, Calif., 1964

[12] E. Sevin, On the elastic bending of columns due to dynamic axial forces including effects of axial inertia, J. Appl. Mech. 27, 125-131 (1960)

[13] R. W. Dickey, Free vibrations and dynamic buckling of the extensible beam, J. Math. Anal. Appl. 29, 443-454 (1970)

[14] E. L. Reiss, Column buckling-an elementary example of bifurcation, Bifurcation theory and nonlinear eigenvalue problems, Benjamin, New York, 1969, pp. 1-16

[15] J. J. Stoker, Nonlinear vibrations in mechanical and electrical systems, Interscience, New York, 1950 